\title{
Inkjet Printed LED based pH Chemical Sensor for Gas Sensing
}

\author{
Martina O'Toole $^{\mathrm{a} *}$, Roderick Shepherd ${ }^{\mathrm{b}}$, Gordon G. Wallace ${ }^{\mathrm{b}}$ and Dermot Diamond ${ }^{\mathrm{a}}$ \\ ${ }^{a}$ CLARITY: Centre for Sensor Web Technologies, National Centre for Sensor Research, \\ School of Chemical Sciences, Dublin City University, Dublin 9, Ireland. \\ ${ }^{\mathrm{b}} \mathrm{ARC}$ Centre of Excellence for Electromaterials Science, Intelligent Polymer Research \\ Institute, AIIM Facility, Innovation Campus, University of Wollongong, Squires Way, \\ Fairy Meadow, NSW 2519, Australia.
}

\begin{abstract}
Predictable behaviour is a critical factor when developing a sensor for potential deployment within a wireless sensor network (WSN). The work presented here details the fabrication and performance of an optical chemical sensor for gaseous acetic acid analysis, which was constructed using inkjet printed deposition of a colorimetric chemical sensor. The chemical sensor comprised a $\mathrm{pH}$ indicator dye (bromophenol blue), phase transfer salt tetrahexylammonium bromide and polymer ethyl cellulose dissolved in 1-butanol. A paired emitter-detector diode (PEDD) optical detector was employed to monitor responses of the colorimetric chemical sensor as it exhibits good sensitivity, low power consumption, is low cost, accurate and has excellent signal to noise ratios. The chemical sensor formulation was printed directly onto the surface the emitter LED, and the resulting chemical sensors characterised with respect to their layer thickness, response time and recovery time. The fabrication reproducibility of inkjet printed chemical sensors in comparison to drop casted chemical sensors was investigated. Colorimetric chemical sensors produced by inkjet printing, exhibited an improved reproducibility for the detection of gaseous acetic acid with a relative standard deviation of $5.5 \%$ in comparison to $68.0 \%$ calculated for drop casted sensors $(n=10)$. The stability of the chemical sensor was also investigated through both intra and inter-day studies.
\end{abstract}

\footnotetext{
*Corresponding author: Tel: + +353 1 7007926; Fax: +353 17007995 Email: martina.otoole@dcu.ie
} 
Keywords: Light emitting diode, Inkjet printing, Gas sensor, Colorimetric sensor, Paired emitter-detector diode

\section{Introduction}

The broad reaching implications of global warming throughout the world have placed the spotlight on gas sensing within the scientific community, as monitoring of contaminants in air is critical in understanding and managing potential risks to human health and indeed the environment. Consequently, there is an expanding need for simple, rapid, accurate, cost-effective field-deployable sensors incorporating wireless communication capabilities $[1,2]$. The use of ion-selective electrodes, electrochemical sensors, optical chemical sensors, chemical sensor arrays and biosensors in WSNs has been reviewed recently by Diamond et al. [3, 4] and Janata [5]. The main challenges lie in the production of inexpensive, reproducible and reliable devices with adequate sensitivities and selectivities [6]. All of these criteria must be met for scale-up and mass production of sensors, which ultimately enables widespread field deployment and real-time monitoring. Due to developments within the last decade with regard to the availability of inexpensive optical components such as light emitting diodes (LEDs) and the development of existing chemistries for the detection of a wide variety of gases, optical chemical sensors now lend themselves well to potential field-deployable devices.

Issues with regard to sensitivity and reproducibility still persist however, as a result of the both the fabrication method and optical detection system employed. One way to address the issue of sensitivity is to select the most appropriate optical detection system used in conjunction with the chemical sensing layer. Optical detectors typically employed within optical chemical sensors include LEDs as the light source coupled with a charge coupled device (CCD) [7, 8], light wave multimeter [8] and photodiode [9-11]. Other optical chemical sensors reported have employed a tungsten-halogen light source coupled with a CCD [12] in addition to flatbed scanners [13-15]. While some of these systems can be incorporated into portable handheld devices, they are not suitable for scale-up within a WSN. Previous work carried out by Shepherd et al. [16-20] demonstrated the advantages of employing a paired emitter-detector diode (PEDD) as the detection system of choice within a WSN. A PEDD consists of two LEDs, whereby one serves as the light source 
and the other in reverse bias mode as the light detector. Instead of measuring the photocurrent directly, a simple timer circuit is used to measure the time taken for the photocurrent generated by the emitter LED to discharge the detector LED from $5 \mathrm{~V}$ (logic 1) to $1.7 \mathrm{~V}$ (logic 0 ) to give digital output directly without using an $\mathrm{A} / \mathrm{D}$ converter or operation amplifier [21, 22]. This method achieves excellent sensitivity and signal-tonoise ratio in comparison to the more commonly employed method of coupling an LED to a photodiode [23]. The low cost, size $(3.2 \times 1.6 \mathrm{~mm})$, low power consumption, ease of fabrication and simplicity of the PEDD make it an ideal optical detector for the real time monitoring of target gases and it was successfully deployed within a WSN for the detection of plumes of gaseous acetic acid achieving a limit of detection of $0.03 \mathrm{ppm}$ [19].

The colorimetric $\mathrm{pH}$ chemical sensor formulation employed within the WSN system for the detection of acetic acid was adapted from $\mathrm{pH}$ chemical sensors developed by Crowley et al. which were applied to the real time monitoring of spoilage in fish packaging [9]. Issues of sensor reproducibility were evident in both the WSN [18] and fish packaging [11] manifolds as a result of the manual drop-casting method employed to deposit the chemical sensing layer. Common methods which have typically been employed in the fabrication of chemical sensors include dip-coating [12, 24, 25], drop-casting [16-18, 26], spin-coating [11, 27, 28] and screen printing [9]. Disadvantages associated with these methods can include limited deposition control with regard to film thickness, positioning and patterning capabilities [29]. The emerging technology of inkjet printing for chemical sensor deposition addresses these issues, as the film thickness and consistency is potentially very uniform and hence the resulting sensors should have greatly improved reproducibility in terms of characteristics such as sensitivity, zero setting and drift. Inkjet printing has gradually become a versatile tool for accurately depositing very small quantities (tens of picoliters) of materials at defined positions on the surface of a wide variety of substrates. So far within scientific research, inkjet printing has been mostly applied to the manufacture of polymer light emitting diodes [29-32], deposition of conducting polymers [6, 33-35] and fluorescent nanoparticles [36] and fabrication of biosensors [37, 38]. Inkjet printing offers advantages over other methods of deposition of thin films, such as patterning capability, reduction in waste products, high speed 
production, low cost fabrication, room temperature deposition, printing on large area and flexible substrates [6]. Additional advantages include direct patterning of a substrate surface as inkjet printing does not rely on the use of a specific mask, compared to the multistep process of photolithography [32]. It also allows the operator great freedom with regard to the pattern that can be printed, and the thickness at which the material is deposited. The design template is also simple to create using the associated printer software, compared to the individually designed masks required for screen printing or the complex programming for some other deposition instruments [35]. Also inkjet printing is a non-contact method which, in contrast to contact based methods such as pin spotting systems, has the advantages of reduced contamination and no risk of substrate damage $[32,39,40]$. The prospect of deposition of more than one material is also a definite possibility with multiple ink reservoirs available in standard inkjet printers [35]. By employing inkjet printing it is possible to dispense specific volumes of chemical sensor formulation quickly and accurately, and hence this has huge potential as a suitable scaleup and mass production fabrication method for chemical sensors.

The work presented herein will demonstrate improved fabrication reproducibility than

previously achieved for the detection of gaseous acetic acid, particularly with regard to baseline signal and response slope (sensitivity), by employing inkjet printing as the method through which the chemical sensor will be deposited [18]. As discussed by Reis et al. there are several parameters associated with inkjet printing which will have a significant effect on the film produced [41]. The parameters which were optimised within this work for the direct printing onto an LED surface included temperature, drop spacing, print head height and print design. The effect of film thickness on the resulting sensor's sensitivity, response time and recovery time was investigated, in addition to inter and intra-day reproducibility within a sensor.

\section{Experimental}

\subsection{Chemical Sensor Formulation}

All chemicals used were of reagent grade and purchased from Sigma Aldrich, Dublin, Ireland. Deionised water obtained from a Millipore Milli-Q water purification system 
(Millipore Ireland B.V., Cork, Ireland) was used for all analysis. The colorimetric chemical sensor formulation previously employed for the detection of acetic acid [18] was optimised for drop cast deposition and was therefore not suitable for inkjet printing. The modified chemical sensing polymer for inkjet printing was prepared by preparing a $0.25 \%$ solution of ethyl cellulose (4 cP: Catalogue No. 200646) in 1-butanol. The viscosity of the solution was specifically tailored to enable inkjet printing of the chemical sensor. To $10 \mathrm{~mL}$ of this solvent, $20 \mathrm{mg}$ of the $\mathrm{pH}$ indicator dye bromophenol blue (BPB) sodium salt was added. The phase transfer salt tetrahexylammonium bromide (THABr), which acts as a solid state $\mathrm{pH}$ buffer in the polymer formulation was added to the solution in a 2.5:1 (salt to dye) molar ratio. The solution was then sonicated for approximately 3 hours to ensure full dissolution.

\subsection{Inkjet Printing Optimisation and Fabrication of Sensors}

Inkjet printing of the colorimetric chemical sensor was performed using a Fujifilm Dimatix DMP-2811 printer. $1.5 \mathrm{~mL}$ of the chemical sensor formulation was placed into the printer cartridge using a syringe. Print designs were created using the pattern editor in the Dimatix Drop Manager software. Priming of the printhead with the chemical sensor formulation, in addition to cleaning cycles prior, during and after the printing process were carried out to ensure a high quality, uniform sensor film was achieved.

Several parameters associated with the Fujifilm Dimatix DMP-2811 inkjet printer were optimised specific to the BPB chemical sensor formulation. These included substrate and printhead temperature, drop spacing, print head height and print design. Temperature became an important issue when printing more than one layer, as increasing it ensured each deposited layer was dry before the addition of subsequent layers. An optimum temperature of $45{ }^{\circ} \mathrm{C}$ was determined experimentally, as beyond this temperature excessive drying of the polymer film was observed. In combination with increasing the temperature, allowing sufficient time (5 minutes) between the printing of each layer also aided the process of drying, compensating for the inability to use extreme temperatures. A drop spacing of $30 \mu \mathrm{m}$ was employed as this achieved a high quality film while using a minimum volume of chemical sensor formulation. A print head height of $1.7 \mathrm{~mm}$ was selected as non-uniformity of the film was observed on increasing the head height (i.e. 
the distance between the print head and the substrate). The chemical sensor formulation was printed directly onto the lens surface of the light source which was a 1206 surface mount (3.2 mm x $1.6 \mathrm{~mm}$ ) LED (Radionics, Ireland: Part No. 466-3942) after initially cleaning the surface with ethanol. The printed pattern was designed to cover the entire surface of the LED.

\subsection{Absorbance Measurement using a Paired Emitter-Detector Diode (PEDD)}

The absorbance spectra of BPB in both acidic and basic form (Figure 1) were acquired using a $\mu$ Quant ${ }^{\mathrm{TM}}$ platewell reader (Bio - Tek Instruments, Inc., USA). The BPB in acidic (pH 2) and basic ( $\mathrm{pH} 10)$ form had a $\lambda_{\max }$ at $422 \mathrm{~nm}$ and $617 \mathrm{~nm}$ respectively. The absorbance measurements of printed colorimetric sensing films were acquired employing a paired emitter-detector diode (PEDD) optical detector. The PEDD was constructed as previously reported by Shepherd et al. $[16,18]$ by arranging a pair of facing 1206 orange surface mount LEDs on a $2.0 \mathrm{~mm}$ header. An emitter LED with a $\lambda_{\max }$ at $590 \mathrm{~nm}$ was selected as the light source as the overlap between the absorbing species BPB and the light source provide high sensitivity. The emission spectra of the emitter LED was obtained using an Ocean Optic spectrometer (OOIBase 32 ${ }^{\mathrm{TM}}$, Ocean Optics. Inc., Dunedin, USA). The detector used was a red LED with a $\lambda_{\max }$ at $660 \mathrm{~nm}$ (Radionics, Ireland Part No. 466-3914). To facilitate comparison, the emission and absorption spectra were normalized to a 0-1 range by dividing values by the maximum emission and absorbance achieved.

The sensing technique employed with the PEDD sensor was as previously reported [21, 22]. The detector LED in output mode was charged up to $5 \mathrm{~V}$ for $100 \mu$ s and then switched to high impedance input mode. Photons from the emitter LED generate a photocurrent in the reverse biased detector LED, which discharges a capacitance based voltage from an initial value of $5 \mathrm{~V}$ (logic 1$)$ to a preset value of $1.7 \mathrm{~V}$ (logic 0$)$ [21, 22]. The time taken for discharge was measured with a simple comparator and timer circuit. A voltage regulator running from a $9 \mathrm{~V}$ battery was used to provide a stable $+5 \mathrm{~V}$ source to drive the circuit and LEDs. This method achieves excellent sensitivity with the added benefit of eliminating the need for an A/D converter or operational amplifier. The signal 
was captured using HyperTerminal software (Microsoft Inc., USA), saved as a text file and then analysed using MS Excel.

As shown in Figure 2, using a SKC Aircheck Sampler (Model 224-PCXR3: Air-met Scientific, Australia) air was drawn in through a 50:50 acetic acid to deionised water solution at a constant flow rate of $2 \mathrm{~L} \mathrm{~min}^{-1}$ for 20 seconds. The switch value was then alternated and air was purged through the system at the same flow rate for 2 minutes.

\section{Results and Discussion}

\subsection{Effect of Layer Thickness on Response Characteristics}

Response characteristics such as the magnitude of the response and the time taken to both respond and recover are essential elements to consider when optimising a chemical sensor. To investigate the effect of layer thickness on response magnitude and response/recovery time chemical sensors with varying numbers of layers were fabricated and tested.

\subsubsection{Effect on Response Magnitude - Peak Height}

PEDD chemical sensors of varying layer thickness were made in triplicate. Each PEDD chemical sensor was then tested by exposing it to gaseous acetic acid for 20 seconds followed by air for 2 minutes. This procedure was repeated 4 times and the results obtained are illustrated in Figure 3. The number of layers printed onto the PEDD was directly proportional to the peak height (change in discharge time, $\mu \mathrm{s}$ ) obtained.

Figure 3 shows a linear increase in the peak height calculated for the detection of gaseous acetic acid with increasing number of chemical sensing layers $\left(R^{2}=0.9967\right)$. As outlined in Table 1 the peak height calculated for 1 inkjet printed layer (ca. $100 \mathrm{~nm}$ ) of chemical sensor was $102 \pm 0.5 \mu \mathrm{s}$, which had a relative standard deviation of $0.5 \%(n=3)$. The peak height achieved for 15 inkjet printed layers was $2327.6 \pm 147.2 \mu \mathrm{s}$. The relative standard deviation calculated was $6.3 \%(n=3)$. This showed a $2181 \%$ increase in the peak height obtained using a 15 layer PEDD in comparison to a 1 layer PEDD. 


\subsubsection{Effect on Response Time}

To investigate the effect of layer thickness on time taken to achieve a response maximum, PEDD chemical sensors of varying layer thickness were tested by this time exposing them to gaseous acetic acid for 2 minutes followed by air for 20 minutes. The increased exposure time to acetic acid and air was sufficient to allow a plateau in signal for both the response and recovery. Figure 4 demonstrates the effect the number of inkjet printed layers on the surface of a PEDD chemical sensor has on the time taken to achieve a maximum response. The percentage response data was determined by calculating the percentage ratio of the response after 1 minute over the response observed at 2 minutes.

As shown in Figure 4 the initial increase in the number of inkjet printed layers has a minimal effect on the percentage ratio of response achieved. The PEDD chemical sensors with inkjet printed layers up to 3 reach on average $99.4 \%$ of the full response after 1 minute. As the number of layers increase from 4 onwards there is a decrease in the percentage of response obtained. The decrease in the percentage response calculated from 1 inkjet printed layer to 15 was $11.5 \%$. Figure 4 demonstrates that while increasing the number of print layers will significantly increase the magnitude of the response observed it will also increase the time necessary to achieve a maximum response. The response at 15 layers still exhibits however, a relatively fast response for a chemical sensor, achieving $88.0 \%$ of the full response after 1 minute.

\subsubsection{Effect on Recovery Time}

The investigation of the effect on recovery time was carried out similarly to that of the effect on response time. PEDD chemical sensors of varying layer thickness were tested by exposing them to gaseous acetic acid for 2 minutes followed by air for 20 minutes. The percentage recovery data was determined by calculating the percentage of the recovery after 2 minutes over the maximum recovery observed at 20 minutes.

Figure 5 shows that the number of inkjet printed layers is indirectly proportional to the percentage of full recovery achieved. A PEDD chemical sensor with 1 inkjet printed layer 
achieves $99.2 \%$ of full recovery to the original baseline after 2 minutes. A linear decrease in the percentage recovery is observed up to 10 layers after which the decrease begins to tail off. There is a $19.8 \%$ decrease in the percentage of chemical sensor recovery from 1 inkjet printed layer to 15 inkjet printed layers. A PEDD chemical sensor with a 15 inkjet printed layer achieves $79.5 \%$ of full recovery after 2 minutes, which

demonstrates a fast recovery time in comparison to typical bulk optical membrane recovery times.

Results obtained for the investigation of the effect of layer thickness on response magnitude, response/recovery time (Figures 3-5 respectively) show that all three factors must be considered when selecting the optimum layer thickness for a chemical sensor. While Figures 4 and 5 both demonstrate that increasing the number of inkjet printed layers increases the time required to achieve a $100 \%$ chemical sensor response and recovery, it is clear that the increase in layer numbers has a greater effect on chemical sensor recovery than on the response. Response and recovery time are important factors in field deployable sensors for real-time monitoring. Should a sensor take to long to respond or recover, it may result in the sensor missing any sudden or momentary fluctuations in the target analyte detection.

\subsection{Fabrication Reproducibility}

Fabrication reproducibility is a critical issue when developing chemical sensors for deployment within a WSN. Issues arising when insufficient reproducibility is obtained include the requirement for individual sensor calibration, non-accuracy and imprecision. Colorimetric gas sensors previously developed by Shepherd et al. [16-18] were fabricated by drop casting $(<5 \mu \mathrm{L})$ the chemical sensor formulation directly onto surface of the surface mount LED using a micropipette. To determine if inkjet printing the chemical sensor formulation directly onto the LED surface would improve fabrication reproducibility in comparison to drop casting the chemical sensor formulation, multiple PEDDs were prepared employing both methods.

\subsubsection{Drop Casted Colorimetric PEDD Chemical Sensors}


To investigate the fabrication reproducibility of drop casted colorimetric chemical sensors, 10 PEDDs were prepared. Approximately $5 \mu \mathrm{L}$ of the chemical sensor formulation was drop cast directly onto the surface of the emitter LED. The chemical sensor was dispensed from a manual micropipette. On examination of the LED under a microscope it was evident that a non-uniform film was achieved employing this method. In addition it was also noted that due to the small surface area of the LED (3.2 $\mathrm{mm} \times 1.6$ $\mathrm{mm}$ ) it was difficult to position the sensor drop in exactly the same location every time, which would inevitably lead to variability within a batch.

Figure 6A illustrates the variability between 10 drop casted colorimetric PEDD chemical sensors. The troughs in Figure 6A and 6B represent the exposure of the colorimetric PEDD chemical sensors to gaseous acetic acid and the peaks represent the recovery of the sensors in the presence of air purged through the system. Figure 6A demonstrates that there are significant discrepancies with regard to both the baseline of each individual colorimetric PEDD chemical sensor and the response magnitude. The mean peak height $(\mu \mathrm{s})$ and standard deviation calculated for each individual colorimetric PEDD chemical sensor (Listed PEDD 1 - 10) outlined in Table 2 were determined from multiple injections of gaseous acetic acid $(n=4)$. The relative standard deviation within an individual drop casted PEDD chemical sensor was comparable to those calculated within an individual inkjet printed PEDD chemical sensor (Table 2). The mean peak height $(\mu \mathrm{s})$ obtained for the 10 drop casted colorimetric PEDD chemical sensors was 418.2 \pm 284.4 $\mu$ s. The relative standard deviation calculated was $68 \%$, which would reinforce the variations of the colorimetric PEDD chemical sensors observed under a microscope. Reproducibility within individual colorimetric PEDD chemical sensors also varied, with relative standard deviations from 1.0 to $5.7 \%$ (Table 2).

\subsubsection{Inkjet Printed Colorimetric PEDD Chemical Sensors}

As drop casted colorimetric PEDD chemical sensors were not comparable to 1 layer inkjet printed sensors with regard to response magnitude, hence inkjet printed sensors of 7 layers were prepared for the investigation. As shown in Figure 6B the reproducibility of inkjet printed chemical sensors is much better than that of drop casted chemical sensors. 
The data illustrated in Figure 6B has not been baseline adjusted and the mean peak height calculated for the 10 inkjet printed colorimetric PEDD chemical sensors was $976.8 \pm 54.8$ $\mu$ s (Table 2). The relative standard deviation calculated was $5.6 \%$, which is a $91.8 \%$ decrease in the relative standard deviation determined for the 10 drop casted colorimetric PEDD chemical sensors. These figures demonstrate excellent reproducibility in the fabrication of inkjet printed colorimetric PEDD chemical sensors especially when taking into consideration that the data was acquired from a 7 layer sensor. Fabrication reproducibility is improved further when investigating 1 layer colorimetric PEDD chemical sensors. Mean peak height data outlined in Table 1 for 3 x 1 layer colorimetric PEDD chemical sensors was $102.0 \pm 0.5 \mu \mathrm{s}$ which had a relative standard deviation of $0.5 \%$. The results outlined in Table 2 have implications of a highly reproducible fabrication method for colorimetric chemical sensors. The reproducibility of the baseline and sensitivity reduce the need for individual sensor calibration, which in turn makes them ideal for scale up and mass production.

The comparison between drop casted and inkjet printed deposition methods highlights the improved accuracy achieved with inkjet printing, however, relative standard deviations obtained with spin coated sensors reinforce more effectively the advantages of inkjet printing over alternative deposition methods. While the fabrication method of spin coating in comparison to drop casting demonstrates improved reproducibility with regard to film thickness, it still does not match the reproducibility of inkjet printed chemical sensors. Previous work published within our group by Pacquit et al. employing $\mathrm{pH}$ chemical sensors fabricated through spin coating reported the standard deviations associated with this deposition method [10]. Spin coated sensors of various thicknesses (2.6, 1.4 and 1.0 micron) demonstrated relative standard deviations of 5.5\%, $9.6 \%$ and $19.8 \%$ respectively $(n=2)$. Data outlined in Table 2 for inkjet printed chemical sensors of 7 layer thickness (0.7 micron) report relative standard deviations of \%. Although these spin coated reproducibility results show a marked improvement when compared to the relative standard deviations of drop casted sensors (Table 2) they still demonstrate poorer reproducibility than that achieved with inkjet printed $\mathrm{pH}$ chemical sensors.

\subsection{Intra and Inter-day Reproducibility of Inkjet Printed Chemical Sensors}


Stability of a chemical sensor is an important characteristic for field deployment as sensors which loose stability in a relatively short time can introduce false positive/negative readings and hence are not viable for reliable real-time monitoring. To investigate intra and inter-day reproducibility, an individual 10 layer PEDD chemical sensor was exposed to gaseous acetic acid. The same PEDD was used for all experiments to prevent introduction of errors due to chemical sensor fabrication or LED variability.

\subsubsection{Intra-Day Reproducibility}

Figure 7A demonstrates the intra-day reproducibility of a 10 layer PEDD chemical sensor which was exposed to injections of gaseous acid 5 times with 1 hour intervals. As outlined in Table 3 the peak heights calculated demonstrate good reproducibility with a relative standard deviation of $1.5 \%$. The peak height (change in discharge time, $\mu \mathrm{s})$ data obtained from each injection of gaseous acetic acid was plotted against peak number (injection number). The line bar in Figure 7A represents the average peak height of $1170.7 \mu$ s calculated for the detection of gaseous acetic acid. The dots represent how the individual injections deviated from the average peak height calculated.

\subsubsection{Inter-Day Reproducibility}

To investigate the inter-day reproducibility, an inkjet printed PEDD chemical sensor was exposed to injections of gaseous acid 4 times. This was repeated for ten consecutive days employing the same 10 layer PEDD chemical sensor from the intra-day study and experimental conditions. The data obtained for the mean peak height calculated over 10 days is given in Table 3. An increased relative standard deviation of $6.7 \%$ was calculated however this was still lower than the generally accepted limit of $10 \%$. The line bar in Figure 7B represents the average peak height of $1090.4 \mu$ s calculated for the detection of gaseous acetic acid. The dots represent how the daily average peak height deviated from the overall average peak height calculated for 10 days. The error bars represent the standard deviations calculated for the 4 injections which were performed daily. 
The intra and inter-day reproducibility calculated for a 10 layer PEDD chemical sensor highlighted its suitability as a field deployable sensor within a WSN with relative standard deviations of less than $10 \%$ over a period of 10 days.

\section{Conclusion}

The results obtained using the inkjet printed PEDD chemical sensor have demonstrated improved fabrication, intra and inter-day reproducibility than previously reported for the detection of gaseous acetic acid. This was achieved by employing inkjet printing to deposit the chemical sensor as opposed to drop casting, which to date has been one of the common methods of choice to deposit chemical sensing films. Fabrication reproducibility is essential when developing sensors for WSN deployment. Inkjet printed chemical sensors developed within the work presented here demonstrated a staggering $91.8 \%$ decrease in the relative standard deviation calculated for 10 (7 layer) inkjet printed PEDD chemical sensors in comparison to 10 drop casted PEDD chemical sensors. The benefits of inkjet printing are not only the reduction in relative standard deviations within a batch but the removal of the need for individual sensor calibration which ultimately saves time and hence money.

Intra and inter-day reproducibility within an individual (10 layer) PEDD chemical sensor were also investigated. Relative standard deviations of $1.5 \%(n=5)$ and $6.7 \%(n=10)$ were achieved for intra and inter-day reproducibility studies respectively.

The effects of increasing layer thickness were also investigated with respect to response magnitude, time to respond and recover back to baseline. Increasing the number of layers printed resulted in a linear increase in the magnitude of response observed. The response obtained for 15 layer inkjet printed PEDD chemical sensor demonstrated a $2181 \%$ increase in comparison to the response calculated for a 1 layer inkjet printed PEDD chemical sensor. While increasing the layer thickness increased the response magnitude it must be noted that it also increased the time required reach a maximum response and to fully recover. The decrease in the percentage ratio of response observed after 1 minute over the maximum response calculated from 1 inkjet printed layer to 15 was $11.5 \%$. Recovery time observed a similar reduction with a decrease of $19.8 \%$ in the percentage 
of recovery calculated after 2 minutes over the maximum chemical sensor recovery from 1 inkjet printed layer to 15 inkjet printed layers.

The results presented within in this work, demonstrate enormous potential for the use of an inkjet printed PEDD chemical sensor for the purpose of in situ gas monitoring within a WSN. The PEDD offers advantages of sensitivity, low cost, low power consumption, miniaturisation capabilities, precision with excellent signal-to-noise characteristics, in addition to availability in a broad spectral range down into the deep UV (247 $\mathrm{nm}$ to > $1000 \mathrm{~nm}$ ). This paper has demonstrated that inkjet printing offers not only a significant increase in fabrication reproducibility but a multitude of advantages such as reduction in waste volume and cross contamination as it is a non-contact deposition method, precision with regard to exact location of the sensor deposition and patterning capabilities. Surprisingly, even with advantages such as these, the application of inkjet printing of colorimetric chemical sensors is still relatively in its infancy.

The highly reproducible inkjet printing deposition method coupled with the advantages of the PEDD result in optical chemical sensor which can be applied to the detection of a broad spectrum of target analytes.

\section{Acknowledgements}

The authors wish to thank Dr. King Tong Lau and Dr. Stephen Beirne from the National Centre for Sensor Research who contributed to the earlier development of this work. The authors also gratefully acknowledge financial support from Science Foundation Ireland (SFI) under the CLARITY: Centre for Sensor Web Technologies Award (07/CE/1147).

\section{References}

1. K. R. Rogers, Recent advances in biosensor techniques for environmental monitoring, Anal. Chim. Acta 568 (2006) 222-231.

2. P. Kim, J. D. Albarella, J. R. Carey, M. J. Placek, A. Sen, A. E. Wittrig and W. B. McNamara III, Towards the development of a portable device for the monitoring of gaseous toxic industrial chemicals based on a chemical sensor array Sens. Actuators, B 134 (2008) 307-312

3. D. Diamond, F. J. S. de Viteri, J. F. Cassidy, A. P. Doherty, J. G. Vos, T. McCormack, G. Keating, A. J. Killard, B. M. Manning, R. O'Kennedy, B. McCraith, R. J. Forster and H. McCabe, Principles of Chemical and Biological Sensors, John Wiley \& Sons, Inc., New York, 1998. 
4. D. Diamond, S. Coyle, S. Scarmagnani and J. Hayes, Wireless Sensor Networks and Chemo-/Biosensing, Chemical Reviews 108 (2008) 652-679.

5. J. Janata, Principles of Chemical Sensors, Plenum Press, New York \& London, 1989.

6. M. F. Mabrook, C. Pearson and M. C. Petty, An inkjet-printed chemical fuse, Appl. Phys. Lett. 86 (2005) 0135071-0135073

7. J. C. Carter, R. M. Alvis, S. B. Brown, K. C. Langry, T. S. Wilson, M. T. McBride, M. L. Myrick, W. R. Cox, M. E. Grove and B. W. Colston, Fabricating optical fiber imaging sensors using inkjet printing technology: A pH sensor proofof-concept, Biosensors and Bioelectronics 21 (2006) 1359-1364.

8. B. H. Weigl and O. S. Wolfbeis, Sensitivity studies on optical carbon dioxide sensors based on ion pairing, Sens. Actuators, B 28 (1995) 151-156.

9. K. Crowley, A. Pacquit, J. Hayes, K. T. Lau and D. Diamond, A gas-phase colorimetric sensor for the detection of amine spoilage products in packaged fish IEEE Sensors 2 (2005 ) 754-757.

10. A. Pacquit, K. T. Lau, H. McLaughlin, J. Frisby, B. Quilty and D. Diamond, Development of a volatile amine sensor for the monitoring of fish spoliage, Talanta 69 (2006) 515-520.

11. A. Pacquit, J. Frisby, D. Diamond, K. T. Lau, A. Farrell, B. Quilty and D. Diamond, Development of a smart packaging for the monitoring of fish spoilage, Food Chem. 102 (2007) 466-470.

12. T. Grady, T. Butler, B. D. MacCraith, D. Diamond and M. A. McKervey, Optical Sensor for Gaseous Ammonia With Tuneable Sensitivity, Analyst 122 (1997) 803-806.

13. N. A. Rakow, A. Sen, M. C. Janzen, J. B. Ponder and K. S. Suslick, Molecular Recognition and Discrimination of Amines with a Colorimetric Array Angewandte Chemie International Edition 44 (2005) 4528-4532.

14. N. A. Rakow and K. S. Suslick, A colorimetric sensor array for odour visualization Nature 406 (2000) 710-713.

15. M. C. Janzen, J. B. Ponder, D. P. Bailey, C. K. Ingison and K. S. Suslick, Colorimetric Sensor Arrays for Volatile Organic Compounds, Anal. Chem. 78 (2006) 3591-3600.

16. R. L. Shepherd, W. S. Yerazunis, K. T. Lau and D. Diamond, Novel surface mount LED ammonia sensors, IEEE Sensors 2 (2004) 951-954.

17. R. L. Shepherd, W. S. Yerazunis, K. T. Lau and D. Diamond, Low-Cost SurfaceMount LED Gas Sensor, IEEE Sensors 6 (2006) 861-866.

18. R. Shepherd, S. Beirne, K. T. Lau, B. Corcoran and D. Diamond, Monitoring chemical plumes in an environmental sensing chamber with a wireless chemical sensor network, Sens. Actuators, B 121 (2007) 142-149.

19. S. Beirne, B. Corcoran, K.-T. Lau and D. Diamond, Chemical event tracking using a low-cost wireless chemical sensing network, IEEE Sensors (2008) 16151618.

20. J. Hayes, S. Beirne, B. M. Kiernan, C. Slater, K. T. Lau and D. Diamond, Chemical species concentration measurement via wireless sensors, World Academy of Science, Engineering and Technology 34 (2008) 158-162. 
21. M. O'Toole, K. T. Lau and D. Diamond, Photometric detection in flow analysis systems using integrated PEDDs, Talanta 66 (2005) 1340-1344.

22. K. T. Lau, S. Baldwin, M. O'Toole, R. Shepherd, W. J. Yerazunis, S. Izuo, S. Ueyama and D. Diamond, A low-cost optical sensing device based on paired emitter-detector light emitting diodes, Anal. Chim. Acta 557 (2006) 111-116.

23. M. O'Toole, K. T. Lau, R. Shepherd, C. Slater and D. Diamond, Determination of Phosphate using a Highly Sensitive Paired Emitter-Detector Diode Photometric Detector, Anal. Chim. Acta 597 (2007) 290-294.

24. D. A. Nivens, M. V. Schiza and S. M. Angel, Multilayer sol-gel membranes for optical sensing applications: single layer $\mathrm{pH}$ and dual layer $\mathrm{CO} 2$ and $\mathrm{NH} 3$ sensors, Talanta 58 (2002) 543-550.

25. D. A. Nivens, Y. Zhang and S. M. Angel, A fiber-optic $\mathrm{pH}$ sensor prepared using a base-catalyzed organo-silica sol-gel, Anal. Chim. Acta 376 (1998) 235-245.

26. L. Byrne, K. T. Lau and D. Diamond, Monitoring of headspace total volatile basic nitrogen from selected fish species using reflectance spectroscopic measurements of pH sensitive films, Analyst 127 (2002) 1338-1341.

27. N. Leopold, S. Busche, G. Gauglitz and B. Lendl, IR absorption and reflectometric interference spectroscopy (RIfS) combined to a new sensing approach for gas analytes absorbed into thin polymer films, Spectrochim. Acta, Part A 72 (2009) 994-999.

28. J. Spadavecchia, G. Ciccarella, P. Siciliano, S. Capone and R. Rella, Spin-coated thin films of metal porphyrin-phthalocyanine blend for an optochemical sensor of alcohol vapours, Sens. Actuators, B 100 (2004) 88-93.

29. J. Bharathan and Y. Yang, Polymer electroluminescent devices processed by inkjet printing: I. Polymer light-emitting logo, Appl. Phys. Lett. 72 (1998) 26602662.

30. T. R. Hebner, C. C. Wu, D. Marcy, M. H. Lu and J. C. Sturm, Ink-jet printing of doped polymers for organic light emitting devices, Appl. Phys. Lett. 72 (1998) 519-521.

31. H. M. Haverinen, R. A. Myllylä and G. E. Jabbour, Inkjet printing of light emitting quantum dots, Appl. Phys. Lett. 94 (2009) 0731081-0731083.

32. K. Abe, K. Suzuki and D. Citterio, Inkjet-Printed Microfluidic Multianalyte Chemical Sensing Paper, Anal. Chem. 80 (2008) 6928-6934.

33. B. Li, S. Santhanam, L. Schultz, M. Jeffries-EL, M. C. Iovu, G. Sauvé, J. Cooper, R. Zhang, J. C. Revelli, A. G. Kusne, J. L. Snyder, T. Kowalewski, L. E. Weiss, R. D. McCullough, G. K. Fedder and D. N. Lambeth, Inkjet printed chemical sensor array based on polythiophene conductive polymers, Sens. Actuators, B 123 (2007) 651-660.

34. K. Crowley, A. Morrin, A. Hernandez, E. O'Malley, P. G. Whitten, G. G. Wallace, M. R. Smyth and A. J. Killard, Fabrication of an ammonia gas sensor using inkjet-printed polyaniline nanoparticles, Talanta 77 (2008) 710-717.

35. K. Crowley, E. O'Malley, A. Morrin, M. R. Smyth and A. J. Killard, An aqueous ammonia sensor based on an inkjet-printed polyaniline nanoparticle-modified electrode, Analyst 133 (2008) 391-399.

36. S. Magdassi and M. B. Moshe, Patterning of Organic Nanoparticles by Ink-jet Printing of Microemulsions, Langmuir 19 (2003) 939-942. 
37. Y. M. Bae, B.-K. Oh, W. Lee, W. H. Lee and J.-W. Choi, Immunosensor for Detection of Yersinia enterocolitica Based on Imaging Ellipsometry, Anal. Chem. 76 (2004) 1799-1803.

38. T. N. Stewart, B. E. Pierson, R. Aggarwal and R. J. Narayan, Piezoelectric inkjet printing of a cross-hatch immunoassay on a disposable nylon membrane Biotechnol. J. 4 (2009) 206-209.

39. L. R. Allain, D. N. Stratis-Cullum and T. Vo-Dinh, Investigation of microfabrication of biological sample arrays using piezoelectric and bubble-jet printing technologies, Anal. Chim. Acta 518 (2004) 77-85.

40. M. S. Hasenbank, T. Edwards, E. Fu, R. Garzon, T. F. Kosar, M. Look, A. Mashadi-Hossein and P. Yager, Demonstration of multi-analyte patterning using piezoelectric inkjet printing of multiple layers, Anal. Chim. Acta 611 (2008) 8088.

41. N. Reis, C. Ainsley and B. Derby, Ink-jet delivery of particle suspensions by piezoelectric droplet ejectors, J. Appl. Phys. 97 (2005) 0949031-0949036 
Table 1 Data outlining the effect of increasing the number of inkjet printed layers of chemical sensor on the peak height $(\mu \mathrm{s})$ obtained $(n=3)$.

\begin{tabular}{c|c|c}
\hline Layers & Peak Height $(\mu \mathrm{s})$ & RSD $\%$ \\
\hline 1 & $102.0 \pm 0.5$ & 0.5 \\
2 & $214.3 \pm 18.0$ & 8.4 \\
3 & $388.2 \pm 16.6$ & 4.3 \\
4 & $528.3 \pm 38.9$ & 7.4 \\
5 & $612.3 \pm 36.1$ & 5.9 \\
7 & $960.3 \pm 54.6$ & 5.7 \\
10 & $1480.3 \pm 63.0$ & 4.3 \\
15 & $2327.6 \pm 147.2$ & 6.3 \\
\hline
\end{tabular}


Table 2 Comparative data outlining the mean peak height $(n=4)$ and relative standard deviation obtained for the detection of gaseous acetic acid employing 10 drop casted and 10 inkjet printed colorimetric PEDD chemical sensors.

\begin{tabular}{c|c|c|c|c}
\hline & \multicolumn{2}{|c|}{ Drop Casted } & \multicolumn{2}{c}{ Inkjet Printed } \\
\hline PEDD & Peak Height $(\boldsymbol{\mu s})$ & RSD \% & Peak Height $(\boldsymbol{\mu}$ ) & RSD \% \\
\hline 1 & $240.5 \pm 13.7$ & 5.7 & $1020.1 \pm 32.1$ & 3.1 \\
2 & $128.1 \pm 1.6$ & 1.2 & $913.1 \pm 31.7$ & 3.5 \\
3 & $482.8 \pm 17.6$ & 3.7 & $947.7 \pm 2.9$ & 0.3 \\
4 & $185.6 \pm 1.8$ & 1.0 & $950.9 \pm 27.8$ & 2.9 \\
5 & $582.4 \pm 8.8$ & 1.5 & $1051.9 \pm 24.0$ & 2.3 \\
6 & $1081.3 \pm 13.1$ & 1.2 & $1019.4 \pm 24.7$ & 2.4 \\
7 & $224.9 \pm 3.2$ & 1.4 & $906.2 \pm 27.1$ & 3.0 \\
8 & $596.9 \pm 14.6$ & 2.4 & $961.9 \pm 39.4$ & 4.1 \\
9 & $295.9 \pm 7.1$ & 2.4 & $944.1 \pm 5.1$ & 0.5 \\
10 & $363.4 \pm 6.7$ & 1.8 & $1053.8 \pm 24.6$ & 2.3 \\
\hline Mean & $\mathbf{4 1 8 . 2 \pm 2 8 4 . 4}$ & $\mathbf{6 8 . 0}$ & $\mathbf{9 7 6 . 8} \pm \mathbf{5 4 . 8}$ & $\mathbf{5 . 6}$ \\
\hline
\end{tabular}


Table 3 Intra and inter-day reproducibility study of a (10 layer) inkjet printed colorimetric PEDD sensor for the detection of gaseous acetic acid.

\begin{tabular}{c|c|c}
\hline & Intra-day reproducibility $(\mathbf{n}=\mathbf{5})$ & Inter-day reproducibility $(\mathbf{n}=\mathbf{1 0})$ \\
\hline Peak Height $(\mu \mathbf{s})$ & $1170.7 \pm 17.9$ & $1090.4 \pm 72.8$ \\
\hline RSD \% & 1.5 & 6.7 \\
\hline
\end{tabular}




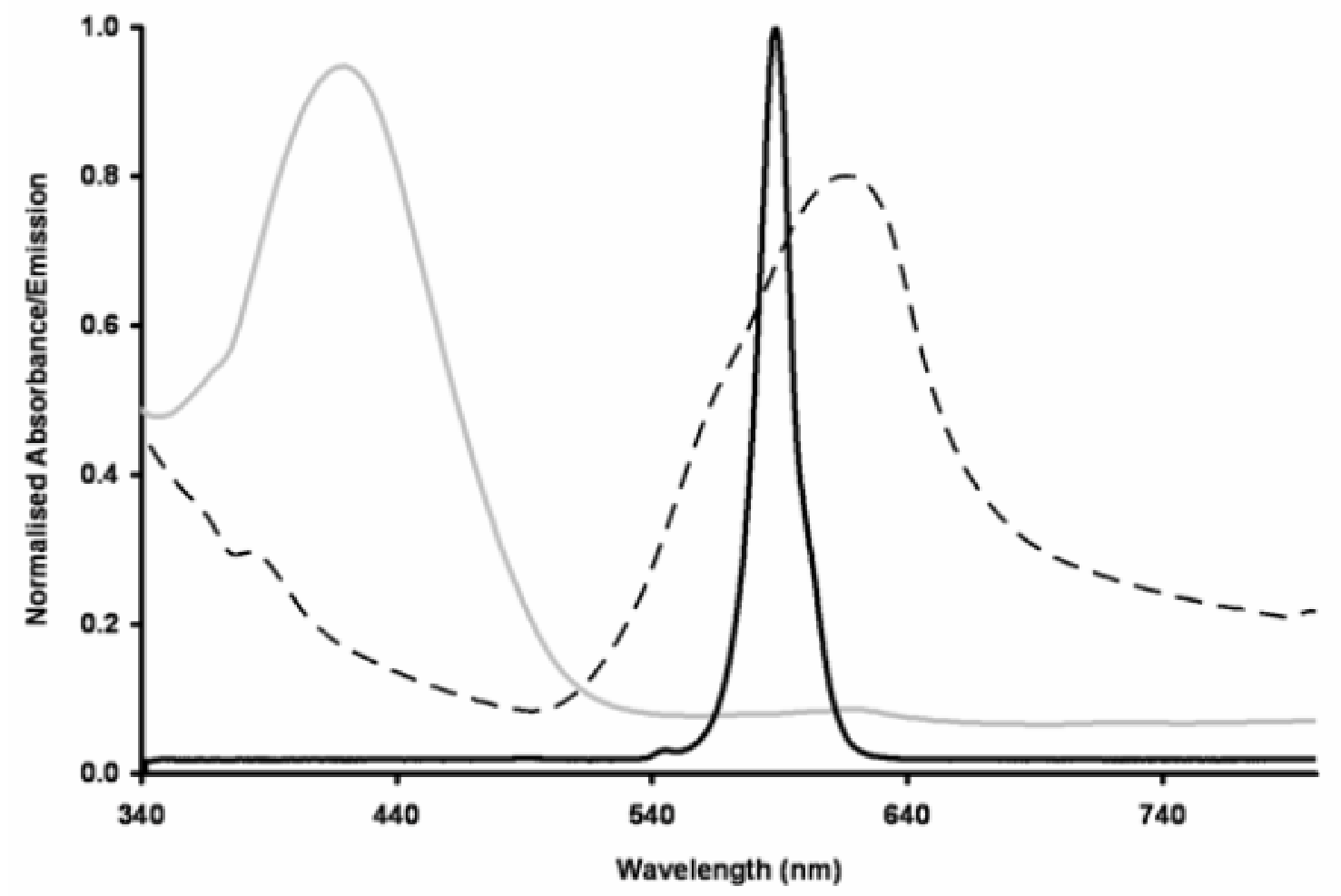

Figure 1 Emission spectrum (bold line) of the emitter LED $\left(\lambda_{\max } 590 \mathrm{~nm}\right)$ and the absorption spectra of BPB (dashed line) at pH $10\left(\lambda_{\max } 617 \mathrm{~nm}\right)$ and (bold grey line) pH $2\left(\lambda_{\max } 422 \mathrm{~nm}\right)$ 


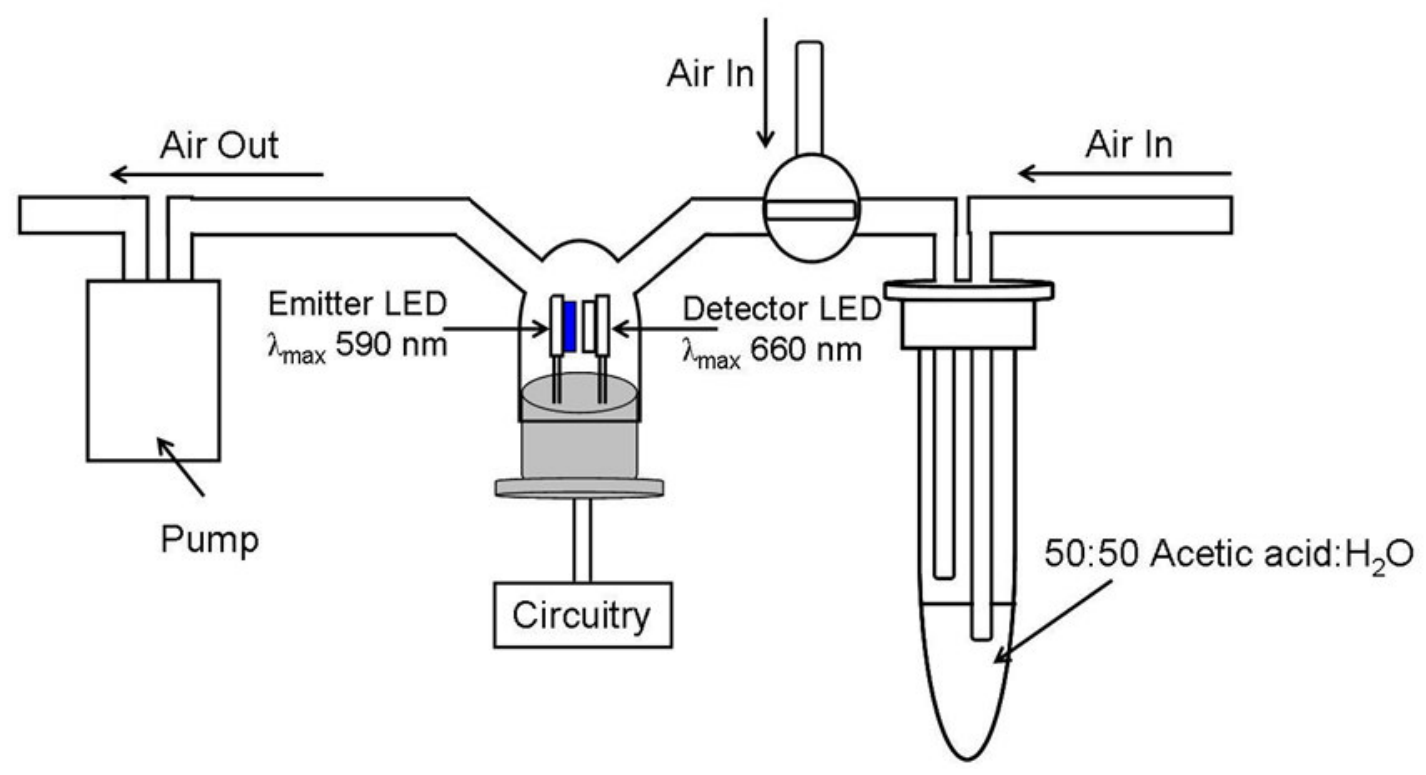

Figure 2 Schematic of the experimental set-up for the detection of gaseous acetic acid 


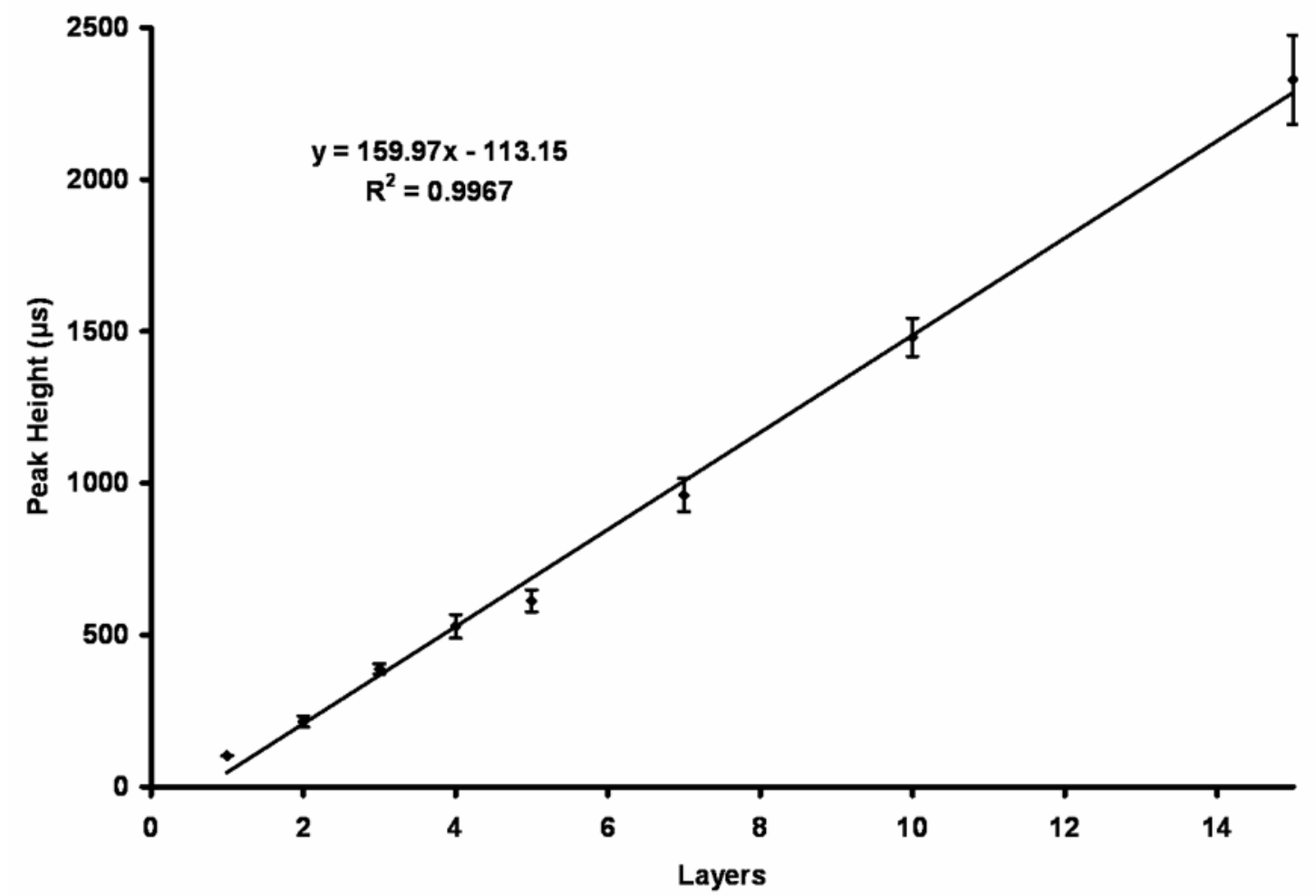

Figure 3 Calibration plot representing the number of inkjet printed layers on the surface of a PEDD sensor versus the peak height $(\mu s)$ obtained. The error bars represent the standard deviations for $n=3$. 


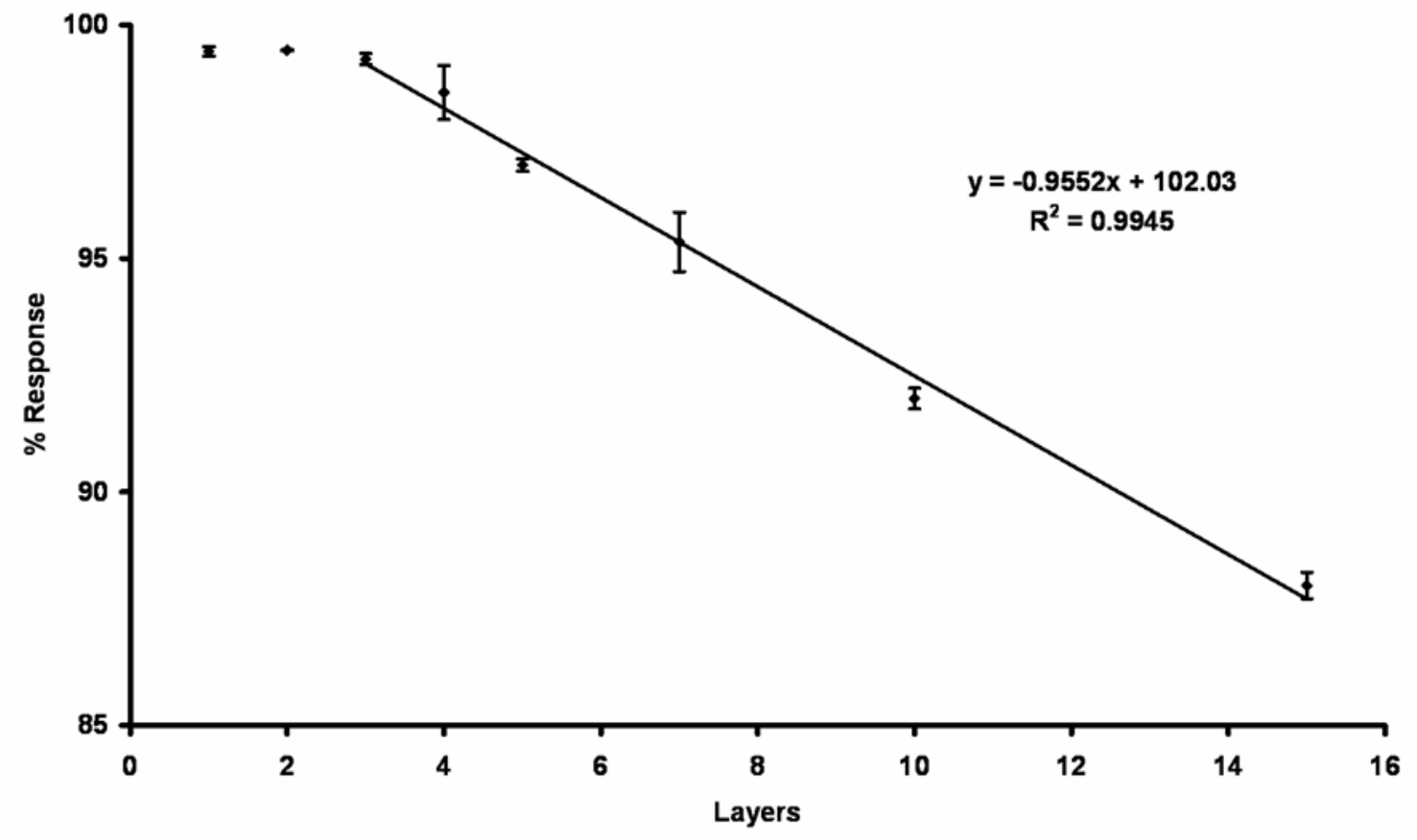

Figure 4 Calibration plot representing the number of inkjet printed layers on the surface of a PEDD chemical sensor versus the percentage of full response achieved after 1 minute. The error bars represent the standard deviations for $n=3$. 


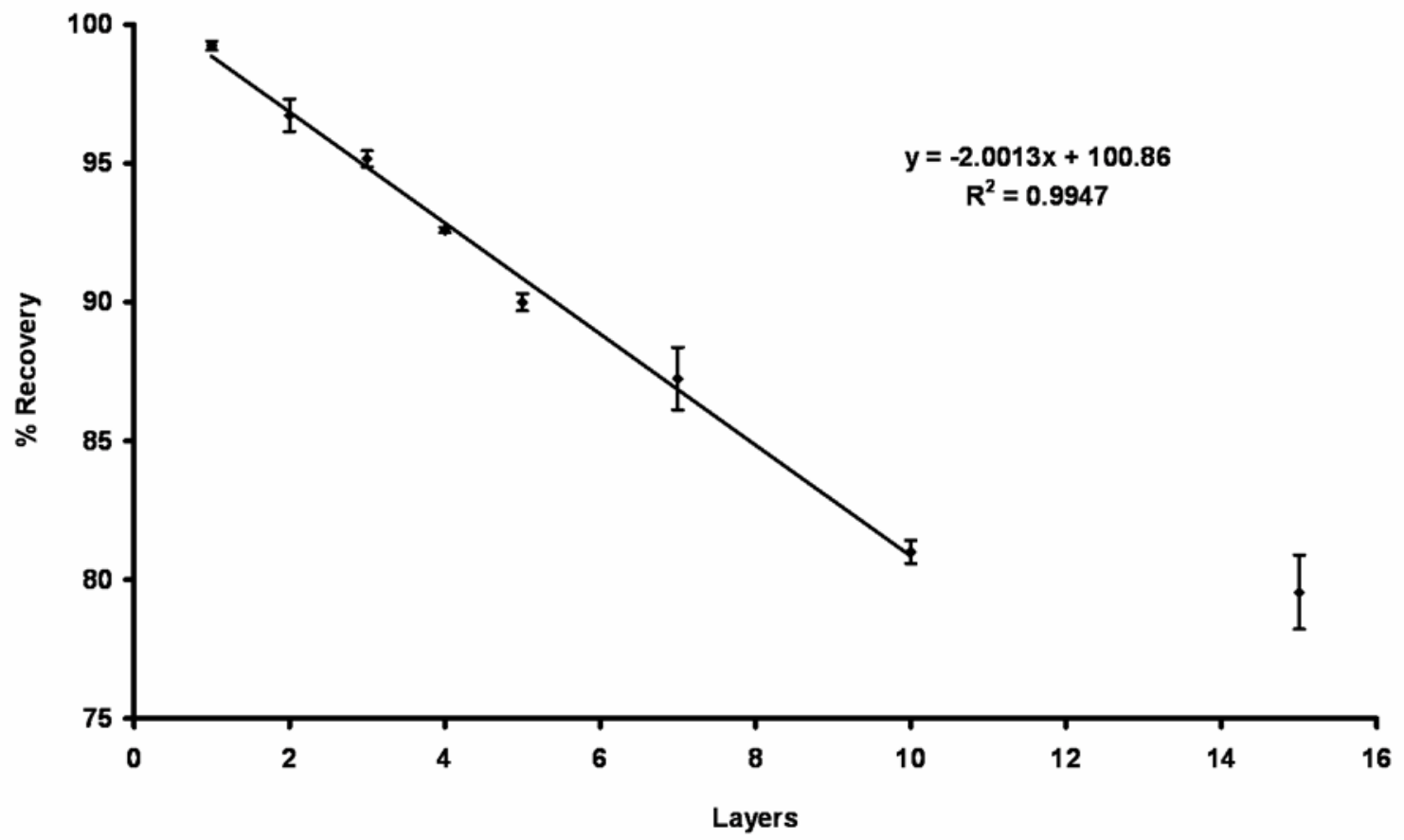

Figure 5 Calibration plot representing the number of inkjet printed layers on the surface of a PEDD sensor versus the percentage of full recovery achieved after 2 minutes. The error bars represent the standard deviations for $n=3$. 

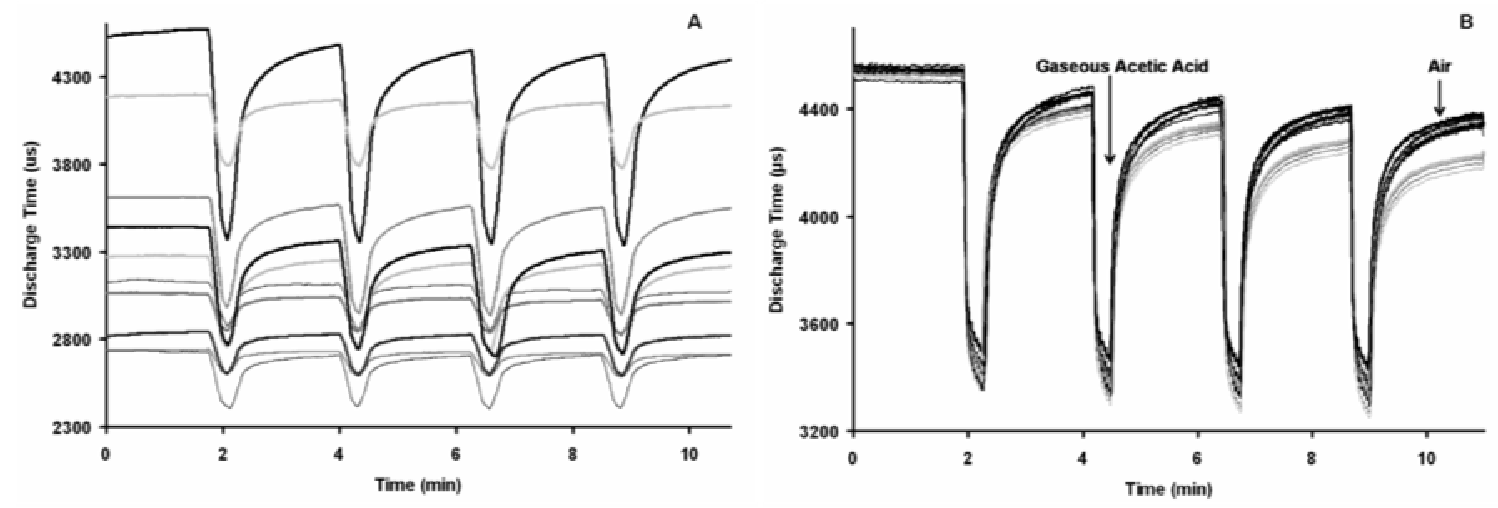

Figure 6 Plot obtained from multiple $(n=4)$ injections of gaseous acetic acid employing 10 (A) drop casted and (B) inkjet printed colorimetric PEDD chemical sensors. 

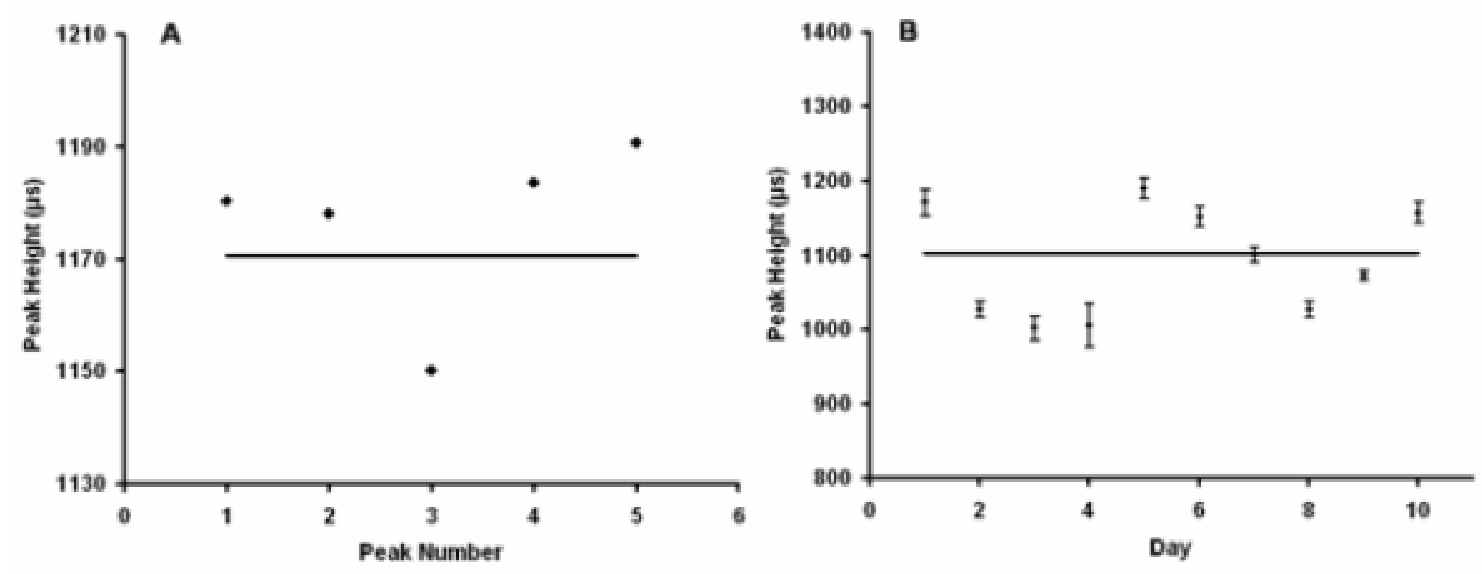

Figure 7 Mean peak height ( $\mu \mathrm{s})$ data for the detection of gaseous acetic acid using a 10 layer PEDD chemical sensor for (A) intra-day $(n=5)$ and (B) inter-day $(n=10)$ reproducibility study. 